\title{
Aptasensor for quantification of leptin through PCR amplification of short DNA-aptamers
}

\author{
Francesca Romana Cavallo, ${ }^{*, \dagger}$ Khalid B. Mirza, ${ }^{*},+, \dagger$ Sara de Mateo, ${ }^{\dagger}$ Konstantin \\ Nikolic, ${ }^{\dagger} \uparrow$ Jesus Rodriguez-Manzano, ${ }^{\dagger, \S}$ and Christopher Toumazou ${ }^{*, \dagger}$ \\ $\dagger$ Centre for Bio-Inspired Technology, Imperial College London, UK \\ $\ddagger$ Dept. of Biotechnology and Medical Engineering, National Institute of Technology, \\ Rourkela, India \\ ISchool of Computing and Engineering, University of West London, UK \\ $\S$ Department of Infectious Disease, Imperial College London, UK
}

E-mail: francesca.cavallo14@imperial.ac.uk; baigm@nitrkl.ac.in; c.toumazou@imperial.ac.uk

\begin{abstract}
Protein quantification is traditionally performed through enzyme-linked immunosorbent assay (ELISA), which involves long preparation times. To overcome this, new approaches use aptamers as an alternative to antibodies. In this paper, we present a new approach to quantify proteins with short DNA aptamers through polymerase chain reaction $(\mathrm{PCR})$ resulting in shorter protocol times with comparatively improved limit of detection. The proposed method includes a novel way to quantify both the target protein and corresponding short DNA-aptamers simultaneously, which also allows to fully characterise the performance of aptasensors. Human leptin is used as a target protein to validate this technique, because it is considered an important biomarker for obesity-related studies. In our experiments we achieved a lowest limit of detection of $100 \mathrm{pg} / \mathrm{mL}$ within less than two hours, a limit affected by the dissociation constant
\end{abstract}


of the leptin aptamer, which could be improved by selecting a more specific aptamer. Because of the simple and inexpensive approach, this technique can be employed for Lab-On-Chip implementations and for rapid 'on-site' quantification of proteins.

\section{Keywords-Aptamers, protein quantification, leptin, qPCR, target-induced disassociation}

Protein detection is traditionally performed through enzyme-linked immunosorbent assay (ELISA),${ }^{1}$ which is commercially available for a wide number of target molecules. Despite being an established method for protein analysis, ELISA suffers from certain disadvantages, the major one being the laborious and time consuming steps in high-resource settings.

Aptamer-based methods are a popular alternative to ELISA, which relies on antibodies. Aptamers are selected through systematic evolution of ligands by exponential enrichment (SELEX), ${ }^{2,3}$ an in-vitro process by which aptamers are selected from a large sequence library based on affinity to the target. Consequently, aptamers present several advantages when compared to antibodies: good affinity and specificity combined with selection through rapid in-vitro synthesis ${ }^{4}$ and simpler workflow. A significant advantage of DNA-based aptamers is the ability to be detected and amplified through polymerase chain reaction (PCR), thus offering theoretical limits of detection of just a few target copies.

In this paper, in order to overcome the limitations of ELISA methods and lab-based aptamer methods, we propose a novel aptasensor for protein quantification involving DNA amplification through PCR. This also includes a novel way to amplify short DNA aptamers, enabling simultaneous quantification of aptamers and leptin through a single quantitave PCR (qPCR) amplification. Moreover, aptamer quantification helps to fully characterise the performance of aptasensors, which is normally evaluated at the end, making optimisation challenging. ${ }^{5}$ The use of short aptamers is a further barrier to assay characterisation, since short sequences cannot be amplified by PCR. Our method overcomes this by indirect amplification of short aptamers. Among the advantages of the proposed method are the low number of reagents needed and the quick preparation not requiring washes and long incu- 
bation times, making it a rapid, low cost and easy assay for protein detection. The absence of magnetic beads as employed in previous works, ${ }^{6}$ ? makes this method a candidate choice for Lab-on-Chip implementations.

Aptamers combined with qPCR amplification have been previously used for detection purposes. Aptasensors involving immobilised aptamers and $\mathrm{qPCR}$ have been developed for ochratoxin $\mathrm{A}^{7}$ and aflatoxin $\mathrm{B} 1 ;^{8}$ while aptasensors based on rolling cycle amplification have been successfully developed for Vibrio parahaemolyticus ${ }^{9}$ and adenosine triphosphate. ${ }^{10}$ However, no aptasensor has been developed for leptin. In this paper, we employ an aptasensor combined with PCR amplification to detect leptin for the first time. But despite being validated on leptin, the present aptasensor technique can be employed to detect any protein target, provided a suitable aptamer exists. Therefore, our work is also a contribution towards general protein detection with aptasensors using NA amplification. Leptin is a small protein involved in metabolism and body weight regulation whose deficiency results in obesity and type 2 diabetes. ${ }^{11}$ Leptin is produced by the adipose tissue and its receptors are expressed mainly in the hypothalamus, where leptin affects the dopamine pathway responsible for appetite and food intake. ${ }^{12}$ Leptin has been proposed for use in therapies for leptin-deficient patients ${ }^{13}$ and is a potential biomarker in obesity management therapies, as leptin resistance leads to obesity ${ }^{14}$ and since leptin levels vary in relation to dieting therapies. ${ }^{15}$ Additionally, leptin is a biomarker for estimating the risk of ischemic heart disease in type 2 diabetes patients, ${ }^{16}$ and to differentiate between Crohn's disease and ulcerative colitis. ${ }^{17}$

Current methods to detect leptin involve the use of antibodies, and aptamer-based techniques have never been used to quantify leptin before. ELISA ${ }^{18}$ is the most widely used method to detect leptin in serum and plasma; other immunoassays for leptin have been developed using optical ${ }^{19,20}$ and electrochemical ${ }^{21-24}$ techniques. The availability of a quick assay for leptin quantification has research and clinical significance: a rapid detection method can accelerate research into the physiological mechanisms through which leptin regulates energy balance and immune response, which still remain poorly understood. ${ }^{11}$ Moreover, 
given that leptin levels change at a greater rate compared to weight loss under an energy restricted diet, ${ }^{12}$ monitoring leptin concentration during dieting therapies can provide personalised insights on the success of the intervention. Developing a quantitative protein test for point-of-care testing can improve healthcare in many applications and transform the area of proteomics. Given the potential to perform label-free nucleic acid amplification and with further optimisation, the proposed method can be translated into a point-of-care platform with high sensitivity and portability, and reduced costs, complexity and time-to-result, as demonstrated in previous works. ${ }^{25,26}$

\section{Material and Methods}

\section{Materials and reagents}

Leptin was purchased from Thermo-Fisher as part of the Invitrogen Leptin Human ELISA Kit (Catalog no. KAC2281); lysozyme was also purchased from Thermo-Fisher. A leptin binding buffer (50 mM Tris $\mathrm{pH} 8.0,140 \mathrm{mM} \mathrm{NaCl}, 1 \mathrm{mM} \mathrm{MgCl} 2(\mathrm{pH} 7.5)$ ) is used in the sample incubation step. The chemicals used to prepare the buffers where purchased from Sigma-Aldrich. The oligonucleotide sequences were purchased from IDT DNA Technologies. Primers were synthesised with standard desalting, while the leptin adaptor with HPLC purification. The leptin aptamer is taken from Ashley and $\mathrm{Li}^{27}$. The adaptor is designed to have 100bp from the Green Fluorescent Protein (GFP) gene with an extra 26bp for aptamer binding, while the complex sequence is designed to contain the forward adaptor sequence and the reverse aptamer sequence. The adaptor forward and reverse primers are taken from Neuzil et al. ${ }^{28}$. All the oligonucleotide sequences can be found in the Supporting Information

file. For qPCR, we used the SYBR Green FastStart Essential DNA Green Master from Roche LifeScience. 


\section{Protocol}

The proposed method is illustrated in Figure 1a, b and c. Adaptors are incubated at $95{ }^{\circ} \mathrm{C}$ for 5 minutes to disrupt the secondary structures and then mixed with aptamers, in a 1:1 $(\mathrm{v} / \mathrm{v})$ ratio. After mixing gently, they are incubated at $37{ }^{\circ} \mathrm{C}$ for 15 minutes to generate the aptamer-adaptor complex by hybridization. The adaptor-aptamer complexes are mixed with leptin and leptin binding buffer in a 1:1:8 (v/v) ratio and incubated at $37^{\circ} \mathrm{C}$ for 30 minutes.

This protocol relies on the amplification of the aptamer-adaptor complexes elongated by polymerase (Figure 1b), which is inversely proportional to the leptin concentration. The method for amplification of the elongated complexes was developed to optimise a previous version of this assay which employed magnetic beads (see Supporting Information), but was found to be inferior to the presented protocol. After incubation with leptin, $5 \mu \mathrm{L}$ of DNA template are mixed with $10 \mu \mathrm{L}$ of FastStart Essential DNA Green Master and $3 \mu \mathrm{L}$ of DRf water and incubated at $60^{\circ} \mathrm{C}$ for 10 minutes to allow the elongation of the sequences forming the complexes (Figure 1b). Finally, $2 \mu \mathrm{L}$ of forward and reverse primer mix at $5 \mu \mathrm{M}(10 \mathrm{x})$ are added to obtain a $20 \mu \mathrm{L}$ reaction. The optimal primer amount of $0.5 \mu \mathrm{M}$ was found by titrating primer concentrations between $0.3 \mu \mathrm{M}$ and $0.7 \mu \mathrm{M}$. The forward primer anneals to the adaptor part of the complex, while the reverse primer to the aptamer part, which allows for complex amplification without amplifying free adaptors (Figure 1c).

The PCR cycling conditions were pre-incubation at $95^{\circ} \mathrm{C}$ for 600 s and 45 cycles of 3-step amplification at $95{ }^{\circ} \mathrm{C}$ for $10 \mathrm{~s}$, at $60{ }^{\circ} \mathrm{C}$ for $10 \mathrm{~s}$ and $72^{\circ} \mathrm{C}$ for $10 \mathrm{~s}$. Fluorescence measurements were taken after each annealing step.

\section{Assay validation}

The assay was validated on human saliva. Samples were centrifuged at $15,000 \times \mathrm{g}$ for 10 minutes to remove debris. To remove nucleic acids which could interfere with the assay, the sample was prepared following Xuan et al.. ${ }^{29} 5 \%$ PEI was added to the supernatant 

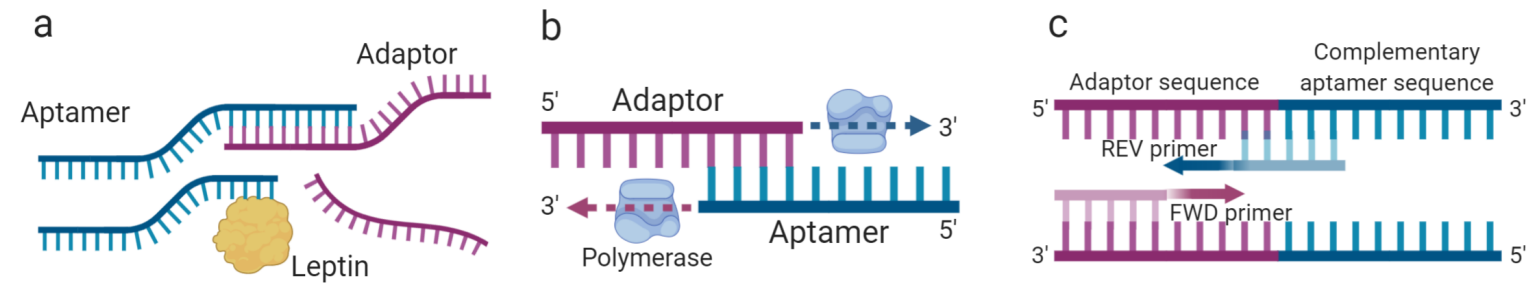

Figure 1: Proposed method for aptamer-based protein quantification. (a) The affinity of the aptamer is higher for leptin than for the adaptor, which gets displaced from the complex as leptin binds to the aptamer. (b) The complexes not displaced by leptin are incubated with polymerase enzyme (see Materials and Methods) to elongate the complexes and create the target DNA for PCR amplification. (c) The elongated complexesinversely proportional to leptin concentration-are amplified with a forward primer binding to the adaptor part of the elongated complex, and a reverse primer binding to the aptamer part of the complex.

to a concentration of $0.5 \%(\mathrm{w} / \mathrm{v})$ followed by incubation for 10 minutes and centrifugation at $15,000 \times \mathrm{g} \mathrm{f}$ for 10 minutes. $474 \mathrm{~g} / \mathrm{l}$ of ammonium sulfate was added to the supernatant with stirring for 20 minutes. After centrifugation at $17,000 \times \mathrm{g}$ for 20 minutes, the pellet was washed three times with ammonium sulfate at $70 \%$ to remove excess PEI and resuspended in DRf water. Assay specificity was assessed by comparing the complex displacement between leptin and lysozyme, a protein present in high concentrations $(\mu \mathrm{g} / \mathrm{mL})$ in saliva. ${ }^{30}$ To measure recovery, saliva samples were spiked with different concentrations of synthetic leptin $(0.1$ $\mathrm{ng} / \mathrm{mL}, 1 \mathrm{ng} / \mathrm{mL}, 10 \mathrm{ng} / \mathrm{mL}$ and $100 \mathrm{ng} / \mathrm{mL}$ ).

\section{Results}

To assess the specificity of the primers, a standard curve were built for the adaptor-aptamer complexes (Figure 2a), which are also used to calculate the leptin concentration from standards during assay optimisation. The primers also had higher specificity to complexes compared to free adaptors (see Supporting Information).

To solve the issues associated to a previous version of this assay employing magnetic beads (for protocol and results, see Supporting Information), to reduce the limit of detection and the assay preparation time, the method presented here was developed without magnetic beads. To optimise the previous assay, a novel method which was developed to indirectly 


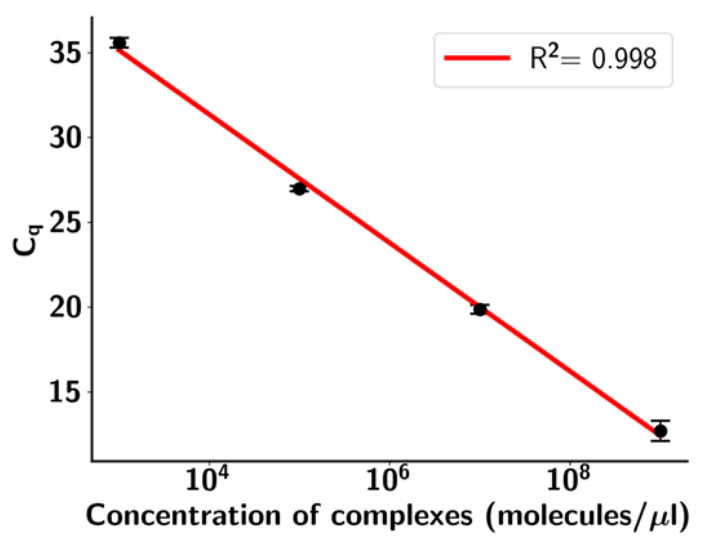

(a) Standard curve for complexes

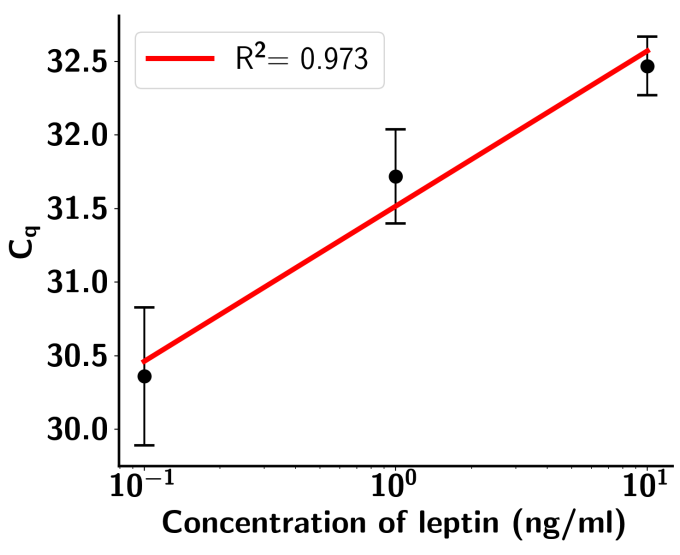

(b) Standard curve for leptin controls.

Figure 2: Standard curves for the quantification of aptamer-adaptor complexes (a) and leptin (b).

quantify aptamers is now used to quantify leptin, which is inversely proportional to the number of leftover complexes. Including a sample for the amount of initial complexes formed after the first hybridization between aptamer and adaptor allows to adjust for the inefficiency of complex formation due to different ambient conditions: this is done in the same assay thus eliminating the need for further reagents. Without magnetic beads, the preparation time is reduced from two hours to less than one hour. Aptamers and adaptors were incubated $37^{\circ} \mathrm{C}$ for 15 minutes. The formed complexes are then incubated with leptin in concentration 10 $\mathrm{ng} / \mathrm{mL}, 1 \mathrm{ng} / \mathrm{mL}$ and $0.1 \mathrm{ng} / \mathrm{mL}$. Amplification curves for leptin concentrations are shown in Figure 3.

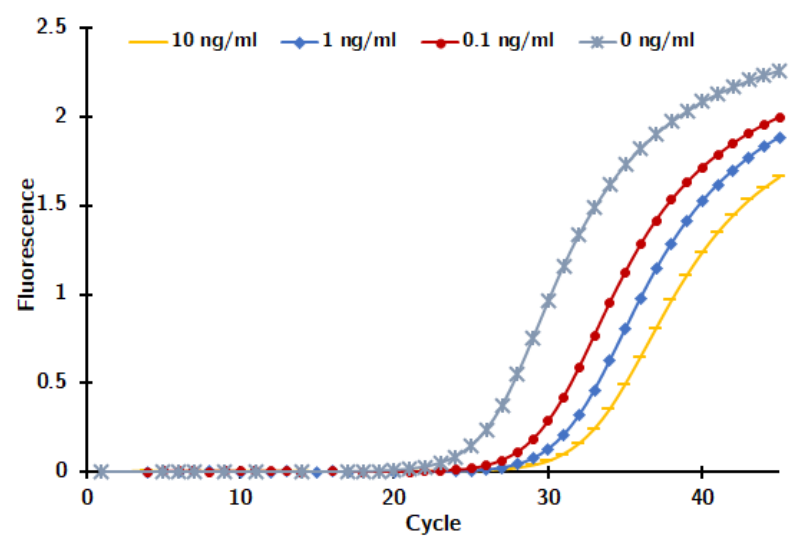

Figure 3: Amplification of leptin standards in concentrations of 10, 1 and $0.1 \mathrm{ng} / \mathrm{mL}$. A negative control with no leptin $(0 \mathrm{ng} / \mathrm{mL})$ is included for calibration purposes. 
The complex formation efficiency greatly affects the performance of the assay, as it determines the amount of complexes available for leptin displacement. The concentration of aptamer and adaptor was optimised by testing the 16 combinations of $330 \mathrm{nM}, 3.3 \mathrm{nM}$, $330 \mathrm{pM}, 3.3 \mathrm{pM}$. When a ratio greater than 2:1 (adaptors:aptamers) is kept, all aptamers form complexes; since the amount of complexes is proportional to the amount of aptamer and adaptor, the optimal concentration was chosen as $330 \mathrm{nM}$ and $3.3 \mathrm{nM}$ for adaptors and aptamers respectively. Detailed results are presented in the Supporting Information.

The choice of buffer influences how well complexes are formed and retained. The leptin binding buffer, TGK buffer (25 mM Tris pH8, $192 \mathrm{mM}$ glycine, $5 \mathrm{mM} \mathrm{K}_{2} \mathrm{HPO}_{4}{ }^{27}$ ) and Guo buffer (10 mM Tris, $120 \mathrm{mM} \mathrm{NaCl}, 5 \mathrm{mM} \mathrm{KCl}, 20 \mathrm{mM} \mathrm{CaCl}_{2} \mathrm{pH} 7.0^{8}$ ) were tested: TGK buffer and distilled water produced the best results in terms of complex retention, but with the leptin binding buffer higher repeatability was achieved. The $\mathrm{pH}$ of the leptin binding buffer influenced the ability for leptin to displace the complexes. Nine $\mathrm{pH}$ values from 2.5 to 8.3 were tested, with 2.5 and 3.0 producing better linearity and separation between the standard concentrations (results shown in the Supporting Information). To increase complex formation, we tried two rounds of incubation to improve the hybridization between adaptors and aptamers. The first round of incubation was done between aptamers and adaptors at $37{ }^{\circ} \mathrm{C}$ for 15 minutes. For the second round, an aliquot from round 1 and an aliquot of adaptors were mixed in equal volumes and incubated again at $37{ }^{\circ} \mathrm{C}$ for 15 minutes. The second round only slightly increased the amount of complexes formed. Incubation times do not affect the performance of the assay: aptamer, adaptor and leptin were incubated with TGK for 10, 30, 45 and 60 minutes at $37^{\circ} \mathrm{C}$ and the amount of complexes was constant regardless of the incubation time. Results from these extra analyses can be found in the Supporting Information.

The inter-assay variability is shown in Figure 4. In $40 \%$ of the experiments, the control sample $(0 \mathrm{ng} / \mathrm{mL})$ showed later amplification compared to the leptin standards; such assays were marked as invalid and discarded. This indicates that ambient conditions strongly affect 
the assay performance, and that a standard curve with negative control should be always included for reliable quantification.

The specificity of the assay was assessed through interference with lysozyme, shown in Figure 5. For fixed leptin concentration $(62.5 \mathrm{pM})$, the assay did not respond to varying concentrations of lysozyme, as the variation in fluorescence from the reference point is significantly lower than for varying concentrations of leptin with fixed lysozyme concentration (62.5 pM).

The assay was validated on human saliva spiked with 1, 10 and $100 \mathrm{ng} / \mathrm{mL}$ of leptin standards. A control sample of saliva without added leptin was included to adjust for the influence of leptin already present in the sample. Table 1 shows that while there is a 3cycle difference between 100 and $1 \mathrm{ng} / \mathrm{mL}$, recovery is poor due to the late amplification of the control sample $(0 \mathrm{ng} / \mathrm{mL})$. Later amplification of the unspiked saliva sample compared to the $0.1 \mathrm{ng} / \mathrm{mL}$ standard indicates a suboptimal specificity of the aptamer for leptin, as complexes are displaced by interfering proteins present in the saliva sample.

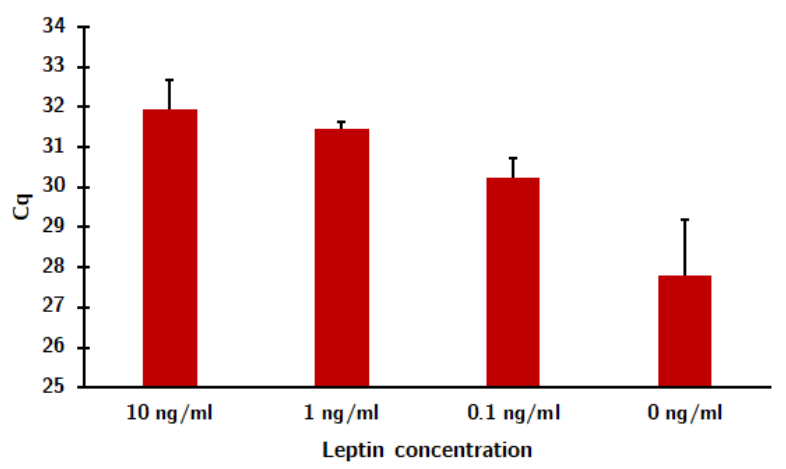

Figure 4: Repeatability of assay for leptin standards. The error bars represent the standard error.

Table 1: Recovery and interference experiment for leptin in human saliva sample.

\begin{tabular}{|c|c|c|c|c|}
\hline Sample & Spiked concentration $(\mathrm{ng} / \mathrm{mL})$ & $\mathrm{Cq}$ mean $\pm \mathrm{SE}$ & Detected concentration $(\mathrm{ng} / \mathrm{mL})($ mean $\pm \mathrm{SE})$ & Recovery \\
\hline \multirow{5}{*}{ Human saliva } & 100 & 47.27 & 3.63 & $4 \%$ \\
\hline & 10 & $43.98 \pm 0.53$ & $5.36 \pm 0.39$ & $54 \%$ \\
\hline & 1 & $42.76 \pm 1.7$ & $0.4 \pm 0.3$ & $40 \%$ \\
\hline & 0.1 & 30.47 & 0 & $0 \%$ \\
\hline & 0 & $41.13 \pm 1.69$ & $\mathrm{~N} / \mathrm{A}$ & $\mathrm{N} / \mathrm{A}$ \\
\hline
\end{tabular}




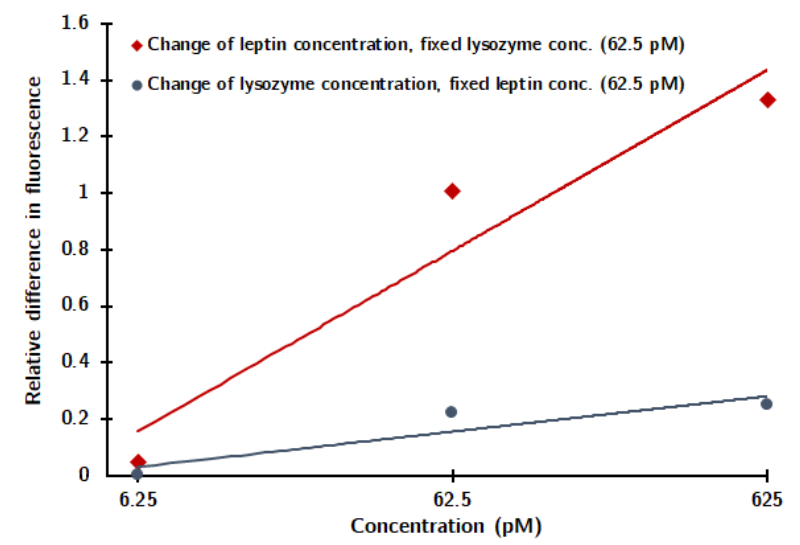

Figure 5: Comparison of the response of the assay to varying concentrations of leptin $(6.25,62.5,625 \mathrm{pM})$ with added lysozyme $(62.5 \mathrm{pM})$, and to varying concentrations of lysozyme $(6.25,62.5,625 \mathrm{pM})$ with added leptin $(62.5 \mathrm{pM})$. The reference points at $6.25 \mathrm{pM}$ have been shifted slightly apart along the y-axis for better visibility.

\section{Discussion}

Aptamers exhibit promising advantages over antibodies for detection of molecular targets: smaller molecular size, ability to select aptamers under in vitro conditions and greater tolerance to room temperature (including reversibility of denaturation due to temperature). A major advantage of nucleic acid-based aptamers is the possibility of amplification through qPCR, thus resulting in very low limits of detection.

Despite these advantages, commercial aptamer detection kits are still not widely available and a lot of the work involving aptamer-based detection is still in research. There are multiple reasons behind this. While aptamers have a clear edge over antibodies in terms of storage, preparation and methodology, comparatively, antibodies have better affinity to the target. This has led to challenges in repeatability when aptamers are used. ${ }^{31}$ There have also been suggestions that the performance of antibodies is still better than aptamers if sensitivity, repeatability and stability are combined together. ${ }^{31}$

However, these considerations apply to lab-based environments. For detection in the field or 'on-site', aptamers seem a better choice than antibodies, due to low detection limits, molecular amplification capability and greater robustness to changes in ambient conditions such as temperature. Hence, in this paper, we presented a simplified, quantitative aptamer- 
based sensing (aptasensor) method using DNA amplification and 'on-site' calibration of aptamer.

A barrier to optimisation of aptasensors is the inability to quantify the assay performance at each step of the protocol, as the performance is evaluated at the end by measuring the response of aptamers to the target. ${ }^{5}$ The ability to quantify aptamers allows optimisation of sensors and assays used. When developing the method with magnetic beads (see Supporting Information), we found the need to quantify the amount of aptamers that did not bind to the beads for optimisation purposes. However, given the short sequence (26bp), we could not amplify the aptamer directly and therefore developed a method to quantify them indirectly by hybridization with the adaptor and elongation of the complex into fully dsDNA, as shown in Figure 1. The simplified protocol without magnetic beads presented in this work was developed with the elongated complexes as the target analyte for leptin quantification. This technique is also useful for quantifying short aptamers-especially for optimisation purposesand for out-of-lab, on-site applications, where different ambient conditions can affect the efficiency of complex formation and assays.

We have demonstrated, to the best of our knowledge, the first aptasensor for detection of leptin and compared its performance and characteristics to other sensors in the literature and to a commercially available ELISA (Table 2). Our aptasensor provides an improvement in reaction times and ease of preparation, while also using a very low sample volume.

Conformational changes to the aptamer-adaptor complexes, which are related to leptin concentration in the unknown sample, are detected using two methods: with and without magnetic beads. The first method (with beads) involves amplification of the adaptors displaced from the complexes by leptin binding to the aptamer (Figure S-2). Figure S-3 shows successful amplification of leptin concentrations from $1 \mathrm{ng} / \mathrm{mL}$ to $0.01 \mathrm{ng} / \mathrm{mL}$. An amplification of negative control is also observed, thus limiting the detection range to about $0.01 \mathrm{ng} / \mathrm{mL}$. The second method (without beads) involves amplification of the remaining aptamer-adaptor complexes after interaction with leptin, which are inversely proportional to 
the amount of leptin molecules that have interacted with the complexes (Figure 1). In this case, no amplification of the negative control was observed, suggesting that the detection limit of the aptasensor could be lowered further, provided that the affinity of the aptamer for leptin is high enough to allow repeatability at low target concentrations.

The assay is validated on human saliva spiked with leptin, showing an increasing number of displaced complexes for increasing leptin concentrations (Table 1). As specificity is an important issue in aptasensors, ${ }^{32}$ we evaluated the specificity of our assay by comparing the response to leptin and to lysozyme. As shown in Figure 5, the variation in displaced complexes is greater for varying leptin concentrations than for varying concentrations of lysozyme, which we chose as an interfering protein given the high concentrations in human saliva.

To characterise and understand the performance of the aptasensor, the kinetics of the different reactions are shown below ( $L$ is leptin, $A p$ is aptamer, $A d$ is adaptor). The first reaction to consider is between the DNA aptamer and leptin:

$$
\begin{gathered}
{[L]+[A p] \stackrel{K_{1}}{\rightleftharpoons}[A p L]} \\
K_{1}=\frac{[A p L]}{[A p][L]}=\frac{1}{K_{d, A p}}, \quad K_{d, A p}=1.5 \pm 0.25 \mu M^{27}
\end{gathered}
$$

where, $\mathrm{K}_{d, A p}$ and $\mathrm{K}_{1}$ are the dissociation constant of the aptamer and equilibrium constant of the leptin-aptamer reaction, respectively. The dissociation constant $\mathrm{K}_{d, A p}$ indicates the affinity of the aptamer towards leptin. The second reaction involves formation of complexes between the aptamer and adaptor:

$$
\begin{gathered}
a[A p]+b[A d] \stackrel{K_{2}}{\rightleftharpoons} c[A p A d] \\
K_{2}=\frac{[A p A d]^{c}}{[A p]^{a}[A d]^{b}} \stackrel{a=b=c=1}{=} \frac{[A p A d]}{[A p][A d]}=\frac{1}{K_{d, A d}}, \quad K_{d, A d}=14.3 n M
\end{gathered}
$$

where $a, b, c$ are stoichiometric units of aptamer $([A p])$, adaptor $([A d])$ and aptamer-adaptor 
complexes $([A p A d])$ respectively; and $\mathrm{K}_{d, A d}$ and $\mathrm{K}_{2}$ are dissociation constant and equilibrium constant of the aptamer-adaptor reaction. $\mathrm{K}_{d, A d}$ was estimated by fitting a logistic function to the aptamer-adaptor binding curve (Figure S-1). When leptin is introduced in a test solution containing $[A p A d]$ complexes, leptin will displace the adaptor from the complex and bind to the aptamer if $\mathrm{K}_{2} \ll \mathrm{K}_{1}$. The reaction between unknown concentrations of leptin and complexes, where $x \leq 1$ is the unknown stoichiometric proportion of leptin $([L])$ in relation to amount of complexes, can therefore be modelled by equations 5 and 6 .

$$
[A p A d]+x[L] \stackrel{K_{3}}{\rightleftharpoons} x[A p L]+(1-x)[A p A d]+x[A d]
$$

Assuming all the leptin has reacted with $A p A d$ complexes, we get the equilibrium constant of the whole reaction, which depends on the dissociation constants of aptamer-leptin and of aptamer-adaptor:

$$
K_{3}=\frac{[A p L]^{x}}{[L]^{x}} \frac{[A d]^{x}}{[A p A d]^{x}}=\left[\frac{K_{d, A d}}{K_{d, A p}}\right]^{x}
$$

$\mathrm{K}_{3}$, which determines the affinity of the complex for leptin, is dependant on the proportion $x$ of leptin and the ratio of the dissociation constants $\mathrm{K}_{d, A d}$ and $\mathrm{K}_{d, A p}$. Hence, higher the proportion of leptin in the test sample, higher will be the affinity; similarly, lower $\mathrm{K}_{d, A d}$ will result in greater affinity towards leptin.

The main limitation of this aptasensor is the high dissociation constant of the leptin aptamer. Complexes consisting of an aptamer and a single strand DNA adaptor have been used before with good results attributable to the high specificity affinity of the aptamer to the target molecule. ${ }^{8}$ However in this paper, the aptamer used has comparatively low affinity to leptin (the dissociation constant $\mathrm{K}_{d}$ is about $1.50 \pm 0.25 \mu \mathrm{M}^{27}$ ), which limits the lowest limit of detection of this aptasensor. To improve assay sensitivity, an aptamer with higher affinity to leptin can be selected through in silico post-SELEX, ${ }^{33}$ by which aptamer-protein interactions can be predicted by using machine learning algorithms to create a DNA library from mutations of the original aptamer. The proposed method can be easily modified to work 
with different aptamers, as it would only require the modification of the adaptor sequence and an updated pair of primers.

Higher sensitivity can also be achieved by immobilising aptamers or adaptors on a solid support material and removing excess sequences after each hybridization step; however, this would increase the assay complexity and the preparation time. Sensitivity and specificity can also be improved by implementing a sandwich assay. ${ }^{34}$

Another factor affecting assay performance is the complex stability. The disassociation constant of the complexes is affected by a number of factors, including the length of the hybridisation bases (Figure 1a), since a greater length of the contact between the aptamer and adaptor would render the complexes more stable. Increased complex stability can improve the specificity of the assay, but also compromise the binding of the aptamer with leptin, which only occurs if the complex is displaced. Other factors to consider for an optimal compromise between specificity, sensitivity and assay complexity are incubation temperature, incubation time and solution ionic strength. ${ }^{35}$

The assay was tested on saliva, which highlighted the need for further optimisation to detect leptin in real-world samples. The presence of inhibitors in saliva is the main factor affecting detection accuracy and sensitivity, since several proteins are present in high concentrations - while other proteins such as leptin in much smaller amounts. ${ }^{36}$ The development of an aptamer with higher specificity for leptin could minimise the impact of inhibitors and improve the assay performance in saliva. There are other issues related to the use of saliva as a diagnostics sample such as viscosity and the presence of food debris, which can be tackled by further sample processing.

The stability of the aptamer-DNA has not been considered in previous works on aptasensors, even though the potential loss of complexes, hence aptamers, due to changes in ambient condition is possible in the field. This causes the release of aptamers, loss of DNA adaptors and will undermine the repeatability of results. When an unknown leptin sample is introduced, it may also interact with the free aptamers, which would lead to incorrect reading of 
the leptin concentrations or number of molecules. This problem is addressed in this paper by 'on-site' calibration for the number of complexes formed and lost due to reasons other than the presence of leptin, which is achieved by including a negative control and standard curve in every assay. This will lead to better quantification of leptin and will indicate the 'starting point' and maximum limit of quantification.

Table 2: Comparison of leptin quantification methods. (LoD: limit of detection; HoT: hands-on time; TtR: time to result).

\begin{tabular}{lllllll}
\hline Reference & Assay type & LoD & K $_{d}$ & HoTa & TtR & Sample \\
\hline Imagawa, $1998^{18}$ & ELISA & $0.78 \mathrm{pg} / \mathrm{mL}$ & $83 \mathrm{pM}$ & $\mathrm{N} / \mathrm{A}$ & $15 \mathrm{hrs}+$ & $100 \mu \mathrm{L}$ \\
He, $2015^{19}$ & Chemiluminescent immunosensor & $0.3 \mathrm{pg} / \mathrm{mL}$ & $\mathrm{N} / \mathrm{A}$ & $\mathrm{N} / \mathrm{A}$ & $44 \mathrm{hrs}+$ & $100 \mu \mathrm{L}$ \\
Tanaka, $2013^{20}$ & Waveguide-mode sensor & $100 \mathrm{ng} / \mathrm{mL}$ & $\mathrm{N} / \mathrm{A}$ & $\mathrm{N} / \mathrm{A}$ & $24 \mathrm{hrs}+$ & $400 \mu \mathrm{L}$ \\
Dong, $2014^{21}$ & Electrochemical immunosensor & $30 \mathrm{pg} / \mathrm{mL}$ & $\mathrm{N} / \mathrm{A}$ & $\mathrm{N} / \mathrm{A}$ & $8 \mathrm{hrs}+$ & - \\
Cai, $2019^{22}$ & Electrochemical immunosensor & $0.036 \mathrm{pg} / \mathrm{mL}$ & $\mathrm{N} / \mathrm{A}$ & $\mathrm{N} / \mathrm{A}$ & $78 \mathrm{hrs}+$ & - \\
Chen, $2010^{23}$ & Electrochemical immunosensor & $10 \mathrm{ng} / \mathrm{mL}$ & $\mathrm{N} / \mathrm{A}$ & $\mathrm{N} / \mathrm{A}$ & $13 \mathrm{hrs}+$ & - \\
Ojeda, $2013^{24}$ & Electrochemical immunosensor & $0.5 \mathrm{pg} / \mathrm{mL}$ & $\mathrm{N} / \mathrm{A}$ & $\mathrm{N} / \mathrm{A}$ & $95 \mathrm{~min}+$ & $50 \mu \mathrm{L}$ \\
Commercial ELISA & ELISA & $15.6 \mathrm{pg} / \mathrm{mL}$ & $\mathrm{N} / \mathrm{A}$ & $1 \mathrm{hr} 20 \mathrm{~min}$ & $3 \mathrm{hrs}$ & $10,100 \mu \mathrm{L}-$ \\
Our assay & Optical (qPCR) aptasensor & $100 \mathrm{pg} / \mathrm{mL} \underline{\mathrm{b}}$ & $1.5 \mu \mathrm{M}$ & $10 \mathrm{~min}$ & $1 \mathrm{hr} 45 \mathrm{~min}$ & $10 \mu \mathrm{L}$ \\
Our assay w beads & Optical (qPCR) aptasensor & $10 \mathrm{pg} / \mathrm{mL} \underline{b}$ & $1.5 \mu \mathrm{M}$ & $1 \mathrm{hr}$ & $3 \mathrm{hrs}$ & $10 \mu \mathrm{L}$ \\
\hline
\end{tabular}

a Hands-on time excludes incubation time and optical reading (the qPCR in the proposed methods takes 1 hour), which are included in the time to result. $\underline{\mathrm{b}}$ The LoD is based on the standard curve. $\underline{\mathrm{c}} 10 \mu \mathrm{L}$ for plasma and serum, $100 \mu \mathrm{L}$ for supernatant.

\section{Conclusion}

The simplicity and versatility of aptamers in terms of preparation, storage and methodology makes them ideal candidates for out-of-lab, fast detection of proteins. A significant advantage of aptamers include the ability to form complexes with oligonucleotides which can be used to detect molecular targets at low concentrations.

In this paper, an aptamer based detection method has been presented as a proof-ofconcept. Preliminary results indicate accurate, fast detection of leptin, with time-to-result shorter than commercially available ELISAs. The lowest limit of detection is enough to detect salivary leptin concentrations, ${ }^{37}$ but could be improved if an aptamer with lower $\mathrm{K}_{d}$ is used. Our future is focused on optimising the calibration step and successively translating this work flow on a Lab-On-Chip described earlier ${ }^{38,39}$ to develop an affordable and ultrasensitive 
assay for point-of-care testing. We are currently working on the implementation of the assay presented in this paper as a cartridge ${ }^{40}$ compatible with the device described by Toumazou et al. ${ }^{38}$

\section{Acknowledgement}

This work was supported by the European Research Council Proof-of-Concept Grant (no. 825796), by UK EPSRC grant EP/N002474/1 and by EPSRC Doctoral Training Program.

\section{Supporting Information Available}

Supporting Information Available: The following files are available free of charge: Supplementary information, containing additional results for proposed aptasensor and description of the aptasensor with beads.

\section{References}

(1) Van Weemen, B.; Schuurs, A. Immunoassay using antigen-enzyme conjugates. FEBS Letters 1971, 15, 232-236.

(2) Ellington, A. D.; Szostak, J. W. In vitro selection of RNA molecules that bind specific ligands. Nature 1990, $\underline{346}, 818-822$.

(3) Tuerk, C.; Gold, L. Systematic evolution of ligands by exponential enrichment: RNA ligands to bacteriophage T4 DNA polymerase. Science 1990, 249, 505 LP - 510.

(4) Cass, A. E.; Zhang, Y. Nucleic acid aptamers: Ideal reagents for point-of-care diagnostics? Faraday Discussions 2011, 149, 49-61.

(5) dos Santos, V. C. F.; Almeida, N. B. F.; de Sousa, T. A. S. L.; Araujo, E. N. D.; 
de Andrade, A. S. R.; Plentz, F. Real-time PCR for direct aptamer quantification on functionalized graphene surfaces. Scientific Reports 2019, 9, 19311.

(6) Le, T. T.; Adamiak, B.; Benton, D. J.; Johnson, C. J.; Sharma, S.; Fenton, R.; McCauley, J. W.; Iqbal, M.; Cass, A. E. Aptamer-based biosensors for the rapid visual detection of flu viruses. Chemical Communications 2014, 50, 15533-15536.

(7) Modh, H.; Scheper, T.; Walter, J. G. Detection of ochratoxin A by aptamer-assisted real-time PCR-based assay (Apta-qPCR). Engineering in Life Sciences 2017, 17, 923930.

(8) Guo, X.; Wen, F.; Zheng, N.; Luo, Q.; Wang, H.; Wang, H.; Li, S.; Wang, J. Development of an ultrasensitive aptasensor for the detection of aflatoxin B1. Biosensors and Bioelectronics 2014, 56, 340-344.

(9) Teng, J.; Ye, Y.; Yao, L.; Yan, C.; Cheng, K.; Xue, F.; Pan, D.; Li, B.; Chen, W. Rolling circle amplification based amperometric aptamer/immuno hybrid biosensor for ultrasensitive detection of Vibrio parahaemolyticus. Microchimica Acta 2017, 184, 34773485 .

(10) Wu, F.; Liu, W.; Yang, S.; Yao, Q.; Chen, Y.; Weng, X.; Zhou, X. An aptamer-based ligation-triggered rolling circle amplification strategy for ATP detection and imaging in situ. Journal of Photochemistry and Photobiology A: Chemistry 2018, 355, 114-119.

(11) Zhou, Y.; Rui, L. Leptin signaling and leptin resistance. Frontiers of Medicine 2013, $\underline{7}$, $207-222$.

(12) Mars, M.; De Graaf, C.; De Groot, C. P.; Van Rossum, C. T.; Kok, F. J. Fasting leptin and appetite responses induced by a 4-day 65\%-energy-restricted diet. International

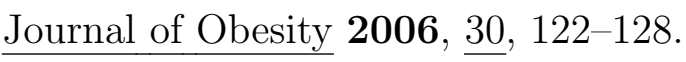


(13) Farooqi, I. S.; O'Rahilly, S. 20 YEARS OF LEPTIN: Human disorders of leptin action. Journal of Endocrinology 2014, 223, T63 - T70.

(14) Friedman, J. M. Leptin and the endocrine control of energy balance. Nature Metabolism 2019, 1, 754-764.

(15) Wadden, T. A.; Considine, R. V.; Foster, G. D.; Anderson, D. A.; Sarwer, D. B.; Caro, J. S. Short-and long-term changes in serum leptin in dieting obese women: effects of caloric restriction and weight loss. The Journal of Clinical Endocrinology \&

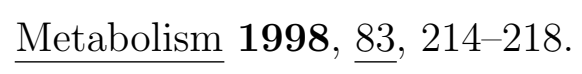

(16) Vavruch, C.; Länne, T.; Fredrikson, M.; Lindström, T.; östgren, C. J.; Nystrom, F. H. Serum leptin levels are independently related to the incidence of ischemic heart disease in a prospective study of patients with type 2 diabetes. Cardiovascular Diabetology $2015, \underline{14}, 62$.

(17) de Carvalho, L. G. F.; Lima, W. G.; Coelho, L. G. V.; Cardoso, V. N.; Fernandes, S. O. A. Circulating Leptin Levels as a Potential Biomarker in Inflammatory Bowel Diseases: A Systematic Review and Meta-Analysis. Inflammatory Bowel Diseases 2020, $\underline{27}, 169-181$.

(18) Imagawa, K.; Matsumoto, Y.; Numata, Y.; Morita, A.; Kikuoka, S.; Tamaki, M.; Higashikubo, C.; Tsuji, T.; Sasakura, K.; Teraoka, H.; Masuzaki, H.; Hosoda, K.; Ogawa, Y.; Nakao, K. Development of a sensitive ELISA for human leptin, using monoclonal antibodies. Clinical Chemistry 1998, 44, 2165-2171.

(19) He, Y.; Sun, J.; Wang, X.; Wang, L. Detection of human leptin in serum using chemiluminescence immunosensor: Signal amplification by hemin/ G-quadruplex DNAzymes and protein carriers by Fe3O4/ polydopamine/ Au nanocomposites. Sensors and Actuators, B: Chemical 2015, 221, 792-798. 
(20) Tanaka, M.; Yoshioka, K.; Hirata, Y.; Fujimaki, M.; Kuwahara, M.; Niwa, O. Design and fabrication of biosensing interface for waveguide-mode sensor. Langmuir $\mathbf{2 0 1 3}, \underline{29}$, $13111-13120$.

(21) Dong, F.; Luo, R.; Chen, H.; Zhang, W.; Ding, S. Amperometric immunosensor based on carbon nanotubes/chitosan film modified electrodes for detection of human leptin. International Journal of Electrochemical Science 2014, 9, 6924-6935.

(22) Cai, J.; Gou, X.; Sun, B.; Li, W.; Li, D.; Liu, J.; Hu, F.; Li, Y. Porous graphene-black phosphorus nanocomposite modified electrode for detection of leptin. Biosensors and Bioelectronics 2019, 137, 88-95.

(23) Chen, W.; Lei, Y.; Li, C. M. Regenerable leptin immunosensor based on protein G immobilized au-pyrrole propylic acid-polypyrrole nanocomposite. Electroanalysis $\mathbf{2 0 1 0}$, $\underline{22,}, 1078-1083$.

(24) Ojeda, I.; Moreno-Guzmán, M.; González-Cortés, A.; Yáñez-Sedeño, P.; Pingarrón, J. M. A disposable electrochemical immunosensor for the determination of leptin in serum and breast milk. Analyst 2013, 138, 4284-4291.

(25) Dirkzwager, R. M.; Liang, S.; Tanner, J. A. Development of Aptamer-Based Point-ofCare Diagnostic Devices for Malaria Using Three-Dimensional Printing Rapid Prototyping. ACS Sensors 2016, 1, 420-426.

(26) De Girolamo, A.; McKeague, M.; Miller, J. D.; Derosa, M. C.; Visconti, A. Determination of ochratoxin A in wheat after clean-up through a DNA aptamer-based solid phase extraction column. Food Chemistry 2011, 127, 1378-1384.

(27) Ashley, J.; Li, S. F. Y. Three-dimensional selection of leptin aptamers using capillary electrophoresis and implications for clone validation. Analytical Biochemistry 2013, $\underline{434}, 146-152$. 
(28) Neuzil, P.; Zhang, C.; Pipper, J.; Oh, S.; Zhuo, L. Ultra fast miniaturized real-time PCR: 40 cycles in less than six minutes. Nucleic Acids Research 2006, 34.

(29) Xuan, J.; Yao, H.; Feng, Y.; Wang, J. Cloning, expression and purification of DNAbinding protein Mvo10b from Methanococcus voltae. 2008,

(30) Jenzano, J. W.; Hogan, S. L.; Lundblad, R. L. Factors influencing measurement of human salivary lysozyme in lysoplate and turbidimetric assays. Journal of Clinical

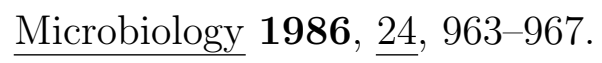

(31) Guo, X.; Wen, F.; Zheng, N.; Saive, M.; Fauconnier, M.-L.; Wang, J. Aptamer-based biosensor for detection of mycotoxins. Frontiers in Chemistry 2020, $\underline{8}$.

(32) Zong, C.; Liu, J. The arsenic-binding aptamer cannot bind arsenic: critical evaluation of aptamer selection and binding. Analytical Chemistry 2019, 91, 10887-10893.

(33) Wang, Q. L.; Cui, H. F.; Du, J. F.; Lv, Q. Y.; Song, X. In silico post-SELEX screening and experimental characterizations for acquisition of high affinity DNA aptamers against carcinoembryonic antigen. RSC Advances 2019, 9, 6328-6334.

(34) Kalra, P.; Dhiman, A.; Cho, W. C.; Bruno, J. G.; Sharma, T. K. Simple methods and rational design for enhancing aptamer sensitivity and specificity. Frontiers in Molecular Biosciences 2018, $\underline{5}, 41$.

(35) Bielec, K.; Sozanski, K.; Seynen, M.; Dziekan, Z.; Ten Wolde, P. R.; Holyst, R. Kinetics and equilibrium constants of oligonucleotides at low concentrations. Hybridization and melting study. Physical Chemistry Chemical Physics 2019, 21, 10798-10807.

(36) Baum, B.; Yates, J.; Srivastava, S.; Wong, D.; Melvin, J. Scientific Frontiers: Emerging Technologies for Salivary Diagnostics. Advances in Dental Research 2011, 23, 360-368.

(37) Gröschl, M.; Rauh, M.; Wagner, R.; Neuhuber, W.; Metzler, M.; Tamgüney, G.; 
Zenk, J.; Schoof, E.; Dörr, H. G.; Blum, W. F., et al. Identification of leptin in human saliva. The Journal of Clinical Endocrinology \& Metabolism 2001, 86, 5234-5239.

(38) Toumazou, C.; Bhimsen, S.; William Green, S.; Harding, P. S.; Sanders, G. H. W.; Wooder, N. J.; Werdich, A. A. Method and apparatus for analysing a biological sample. patent USPTO 20180087097, 2018-03-29.

(39) Toumazou, C.; Shepherd, L. M.; Reed, S. C.; Chen, G. I.; Patel, A.; Garner, D. M.; Wang, C.-J. A.; Ou, C.-P.; Amin-Desai, K.; Athanasiou, P., et al. Simultaneous DNA amplification and detection using a pH-sensing semiconductor system. Nature methods $2013, \underline{10}, 641$.

(40) Cavallo, F. R.; Mirza, K. B.; de Mateo, S.; Nikolic, K.; Jesus, R.-M.; Toumazou, C. 2021 IEEE International Symposium on Circuits and Systems, In Press.

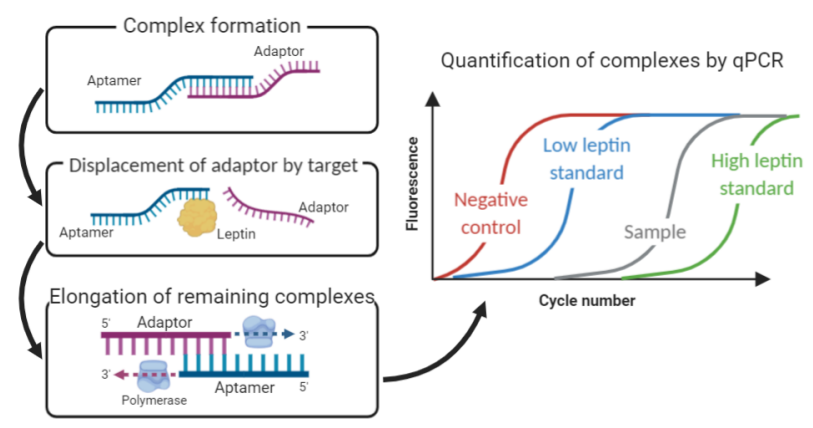

For TOC only 